\title{
Critique du terrain ou terrain critique : de la construction d'un positionnement éthique de recherche dans l'altérité
}

\section{Judicaëlle Dietrich}

\section{(2) OpenEdition}

Journals

Édition électronique

URL : http://journals.openedition.org/cdg/1080

DOI : $10.4000 /$ cdg. 1080

ISSN : 2107-7266

Éditeur

UMR 245 - CESSMA

Référence électronique

Judicaëlle Dietrich, « Critique du terrain ou terrain critique : de la construction d'un positionnement éthique de recherche dans l'altérité », Carnets de géographes [En ligne], 5 | 2013, mis en ligne le 01 janvier 2013, consulté le 21 décembre 2020. URL : http://journals.openedition.org/cdg/1080 ; DOI: https://doi.org/10.4000/cdg.1080

\section{(c) $($ ) $(9)$}

La revue Carnets de géographes est mise à disposition selon les termes de la Licence Creative Commons Attribution - Pas d'Utilisation Commerciale - Pas de Modification 4.0 International. 


\title{
Critique du terrain ou terrain critique : de la construction d'un positionnement éthique de recherche dans l'altérité
}

\author{
JUDICAELLE DIETRICH \\ doctorante en géographie \\ Université de Paris-Sorbonne, Laboratoire ENEC \\ judicaelle.dietrich@gmail.com
}

Mots clés : Démarche empirique et critique, éthique, inégalité, pauvreté, réflexivité, terrain.

\begin{abstract}
Résumé
Du terrain à l'élaboration d'un sujet, la production d'un travail de recherche est issue de choix, de biais méthodologiques, de contextes culturels et situationnels que le chercheur se doit de faire apparaître. C'est l'objet de cet article qui cherche à retranscrire des trajectoires empiriques sur mon terrain de thèse à Jakarta et leurs implications dans la production des informations. À partir d'extraits de mes carnets de terrain, je souhaite montrer pratiques et posture qui participent à la construction de ma démarche soulignant ainsi les enjeux du rapport à l'altérité.
\end{abstract}

Pourquoi travailler sur la pauvreté, et en particulier à Jakarta ${ }^{1} \mathrm{~J}^{\prime}$ ai toujours eu l'impression que ce sujet et ce terrain se sont imposés à moi. Entre un questionnement intérieur qui me taraude depuis plusieurs années et la découverte d'une ville tentaculaire à laquelle je me suis de plus en plus attachée à chaque séjour, il me faut formaliser ce qui dépasse la simple genèse de ma thèse tout en en étant profondément constitutif.

Quand on aborde la bibliographie des études urbaines dans les villes en développement s'intéressant aux inégalités et aux conditions de vie des populations « en marge ", on observe une majorité de travaux portant sur les métropoles d'Amérique latine ou d'Afrique, par rapport à l'Asie. Ces espaces fortement marqués par la colonisation ont fait l'objet d'études dont les héritages marxistes et postmarxistes sont identifiables dans les

\footnotetext{
${ }^{1}$ Titre provisoire de ma thèse: "Inscription spatiale de la pauvreté et production de l'inégalité à Jakarta Indonésie "; Contrat doctoral sous la direction Olivier Sevin, dans le laboratoire ENeC - Espaces Nature et Culture - UMR 8185.
} 
méthodes et les analyses ${ }^{2}$. A l'échelle internationale d'ailleurs, les analyses globales pointent le fait que le phénomène de pauvreté est beaucoup plus rural qu'urbain (bien que ce dernier pose des problèmes de concentration dans des espaces restreints) ; I'Indonésie appuie cette thèse à l'aide de statistiques nationales démontrant que sa capitale n'est pas une ville pauvre et que cette question concerne principalement les zones périphériques du pays, en particulier ses îles dites "sous-développées " ${ }^{3}$. Ainsi, les villes d'Asie du Sud-est ne seraient pas un terrain habituel lorsque l'on interroge ces thématiques et les travaux actuels ont tendance à se concentrer sur les organisations de l'espace à l'échelle régionale en insistant sur les aspects fonctionnels ${ }^{4}$. Jakarta est néanmoins une ville qui a connu des évolutions comparables à de nombreuses autres villes des Suds : capitale coloniale, développement démographique et économique rapide suite à l'Indépendance et ouverture actuelle à la mondialisation néolibérale confirmant la volonté des acteurs dominants (publics et privés) d'insérer la métropole dans le groupe restreint des villes considérées comme globales (Sassen, 2009) ou en passe de le devenir.

Je vais retracer dans ces "carnets de terrain " les trajectoires de mon expérience personnelle et du contexte de construction de mon objet de recherche, en lien avec le terrain et le monde social qu'il implique. Le "terrain » envisagé comme l'espace d'une pratique du chercheur (Volvey, 2003) permet alors d'intégrer une approche réflexive, qu'il me faudra d'abord comprendre afin de faire apparaître le processus d'élaboration de mon sujet. Ensuite, je reviendrai sur quelques expériences de terrain qui révèlent l'intérêt et les limites des analyses dialectiques de la géographie critique dans la compréhension des rapports sociaux observés. Tout cela participe donc à la question plus globale de mon engagement personnel en tant que chercheuse face à des enjeux éthiques, professionnels et personnels mais aussi en tant que personne avec des convictions dont il faut comprendre les implications. L'enjeu ici serait alors de comprendre le rôle de la démarche critique dans la recherche en géographie et ses apports. Elle permet de décrypter les dimensions spatiales des inégalités, les décalages entre discours et pratiques, et ainsi révèle les rapports sociaux en œuvre et les dogmes dont sont issues les actions. Enfin, elle permet de concilier une

\footnotetext{
${ }^{2}$ Les exemples sont nombreux, mais on peut penser aux recherches marquantes de Marianne Morange ou Jean-Fabien Steck respectivement sur l'Afrique du sud et l'Afrique de l'Ouest. Marie Morelle à Antananarivo et Yaoundé a développé la situation des enfants des rues. En Amérique latine, l'importance des mouvements révolutionnaires et les circulations scientifiques majeures participent à la compréhension de la forte présence des études radicales et critiques dans le contexte urbain en particulier. La récupération des conceptions lefebvriennes entre autres a été mise en valeur lors du colloque "La ville compétitive, à quel prix ? "à Nanterre en septembre 2011 et par l'intervention de Claire Revol (2011).

${ }^{3}$ Propos tirés de l'entretien effectué auprès du fonctionnaire du bureau central des statistiques à Jakarta en août 2011. En effet, Jakarta n'aurait que 3,75\% de sa population en dessous du seuil de pauvreté (BPS, 2011), mais ce seuil est établi par ce même bureau à 0,82 dollar par jour, bien en dessous du niveau minimum identifié par la Banque mondiale à 2 dollars, limitant ainsi le nombre de personnes et de ménages concernés.

${ }^{4}$ Ce traitement différencié de l'information par la communauté scientifique a été notamment mis en évidence dans le colloque international Transiter, en février 2012. Cette manifestation scientifique clôturant l'ANR du même nom, cherchait à comparer les dynamiques transnationales et recompositions territoriales en MésoAmérique et en Asie du Sud-est continentale et insulaire. Lors des synthèses des différents ateliers, les différences d'approches et de méthodologies ont été identifiées, relevant les traditions intellectuelles de ces régions du monde qui ont ainsi des conséquences sur les productions scientifiques. Les travaux de Manuelle Franck et de Nathalie Fau, entre autres, font ainsi la part belle aux structures organisationnelles de l'espace supra-régional et aux hiérarchies urbaines qui en découlent. http://transiter.univ-paris-diderot.fr/index.php Cependant d'autres références nuancent ces analyses en particulier les travaux de la Banque Asiatique du Développement (Perception of the poor, 2001, Urban poverty in Asia, 1994).
} 
éthique personnelle avec les exigences méthodologiques d'une recherche en définissant le degré d'engagement du chercheur.

\section{De l'expérience personnelle à la construction d'un objet de recherche : pourquoi travailler sur la question de la pauvreté à Jakarta ?}

Arrivée adolescente, j'ai pu connaître la ville progressivement en apprenant la langue et en y vivant quotidiennement durant une année. Au-delà d'un " choc du tiers-monde ", ce serait plutôt la rencontre inattendue avec un Ailleurs marquée par les incompréhensions et interrogations liées à la nouveauté, la différence et l'inconnu. Les premières impressions au moment de la découverte seraient alors un moyen de contextualiser notre approche, de comparer avec le connu, identifiant finalement tout autant ce qui s'en rapproche de ce qui s'en éloigne. L'évocation de ce qui marque, choque ou interroge permet de confronter notre regard à la différence sans que cela puisse être considéré comme objectif. Et Jakarta m'est apparue avant tout comme incompréhensible, insaisissable, ce que l'on retrouve dans de nombreux textes de scientifiques fraichement débarqués dans la mégapole comme Nigel Barley (anthropologue) ou Christopher Silver (aménageur) qui sont pourtant des chercheurs confirmés habitués à la découverte d'un " autre monde ". Sans que ce soit un cas à part, ce serait ainsi une ville qui s'expérimente, qui doit se vivre avant de se comprendre. Mais cela a permis la création d'un lien personnel avec ce terrain, limitant ma perception de cet espace comme étranger. Ma relation avec Jakarta s'est ensuite précisée grâce à un stage en entreprise associé à un travail de recherche dans la filiale de la multinationale qui gère le traitement et la distribution de l'eau propre dans la partie ouest de Jakarta. Entre anecdote et facteur déclencheur ${ }^{5}$, un événement $m^{\prime}$ a projetée dans mon statut d'Autre :

Au début de mon stage, lors d'une mission de prospection pour l'entreprise, j'ai suivi mon directeur dans un quartier où l'entreprise devait installer de nouveaux réseaux au nord-ouest de la ville, aux limites de la municipalité. Avec une équipe (très identifiable au badge mis en évidence), nous nous sommes rendus à Kalideres. En tenue de bureau, nous voilà en pleine chaleur à arpenter des rues étroites afin de repérer la zone et notamment les espaces libres qui doivent permettre l'installation de compteurs individuels. Comme à chaque fois et malgré les demandes répétées de mon chef (en charge de programme "Water for all ") de se rendre dans de "vrais slums" (il n'utilise jamais le terme français), les agents techniques nous emmènent dans un quartier "ex-illégal", dont la légalité a été reconnue par la municipalité donc, par extension, là où l'entreprise a le droit de desservir la population.

Les heures passent, mon chef prend des photos, les agents prennent des mesures, je prends des notes relevant les activités des populations, le type d'habitat et la forme des rues, l'accès à l'eau apparent... (...) Suite aux observations réalisées pendant quelques heures, nous retournons vers le véhicule de l'entreprise, mais nous nous sommes fortement éloignés. Un agent décide de prendre un raccourci et de passer le long d'un petit canal à ciel ouvert, réceptacle des eaux de pluie et des eaux usées. Le passage se fait de moins en moins praticable, des vêtements qui sèchent "débordent " des

\footnotetext{
${ }^{5}$ Phénomène mis en évidence souvent par les récits de vie analysés selon les méthodes de sociologie.
} 
maisons, un pas mal calculé et ... je tombe (mes notes et mon appareil photo avec). On me hisse hors de l'eau opaque, intégralement trempée, j'y retourne pour aller chercher mon carnet. Cet événement a alors déclenché nombre de réactions: le visage de ceux qui m'entourent s'affole, mon chef, mes collègues mais aussi celui des habitants. Un attroupement se forme en un rien de temps. Tout le monde semble se mobiliser alors que tout va bien, je suis mouillée certes, sale certainement, mais des enfants jouaient le matin dans ce même kali ${ }^{6}$. Je ris de gêne face à l'ampleur que prend ma maladresse. Les voisins m'apportent un verre d'eau en réconfort, mon chef en demande la provenance: "l'eau du réseau municipal monsieur " (l'eau qu'il leur distribue donc) et il refuse qu'on me serve cela et demande qu'on m'apporte une bouteille d'eau fermée. $^{7}$

Ainsi, c'est la réaction des personnes présentes face à ce qui m'apparaissait comme un non événement (ou que je souhaitais le plus discret possible) qui est particulièrement signifiante. Et c'est ce moment qui a participé à ma prise de conscience approfondie des inégalités : ce qui arrivait tous les jours aux locaux ne pouvait voire ne devait pas m'arriver à moi car en plus d'être stagiaire de la compagnie des eaux, j'étais occidentale ${ }^{8}$. Cette opposition radicale entre "eux» et "moi " s'est ainsi imposée. Par ma personne, je représente un grand nombre de différences non négligeables dans les relations qui se tissent entre le chercheur et la population étudiée même dans sa diversité. L'altérité prime dans l'échange. C'est un fait que dans le modèle scientifique positiviste on a tendance à vouloir nier ou à ne pas vouloir voir. Ayant été marquée par ce type d'approche, l'idée d'un point de vue objectif dans la recherche pousse à la négation ou à l'omission du rôle de la personne du chercheur ou de sa subjectivité (Renganathan, 2009). Or la reconnaissance de ce rôle est primordiale pour intégrer la nature située de cette recherche (Pillow 2003). Accepter donc l'altérité et faire accepter ma présence régulière dans leur quotidien n'a pu être possible que par la mise en œuvre de quelques stratégies. Sans prétendre à une intégration, l'idée est de prendre conscience des moyens pour une familiarisation dans leur cadre de vie et ne pas être seulement vue comme l'étrangère potentiellement touriste.

En parallèle, l'enjeu de l'accès aux services urbains élémentaires s'est alors inséré dans la compréhension de la ville et de la société urbaine. Ce stage professionnel de trois mois était conduit dans une démarche de recherche pour mon mémoire de Master $1^{\text {ère }}$ année. L'idée d'une pratique de recherche critique sur les inégalités d'accès à l'eau propre tout en étant responsable dans l'entreprise m'est apparue d'abord contradictoire (pour ne pas dire schizophrène), surtout pour une étudiante peu aguerrie tant au travail dans le privé $q^{\prime}$ 'à la recherche. Il m'a donc fallu m'adapter au fonctionnement de la filiale d'une multinationale et intégrer ses objectifs de rentabilité. La transparence du discours de mon tuteur du fait de mon statut interne à l'entreprise m'a permis de comprendre les implications de sa volonté de distribuer et vendre légalement de l'eau en plus grande quantité aux urbains: derrière l'affirmation de desservir une plus grande partie de la

\footnotetext{
${ }^{6}$ Petit canal ou égout large à ciel à ouvert.

${ }^{7}$ L'eau distribuée par le réseau municipal en gestion privatisée est officiellement reconnue comme eau " propre » et non potable. En pratique, elle est utilisée comme eau de consommation par la majorité des ménages desservis après ébouillantage.

${ }^{8}$ Ces deux aspects faisaient se cumuler les représentations liées aux rapports de domination avec les personnes du quartier
} 
population urbaine à travers leur "mission de service public ", il y a la nécessité de réintroduire les connexions illégales dans le réseau de distribution officiel afin de comptabiliser l'eau consommée, donc d'en tirer un revenu pour l'entreprise. J'ai pu ainsi observer dans les discours de mon employeur, appuyé par de nombreuses ONG, la stigmatisation systématique des réseaux informels de revendeurs d'eau (comparés directement à des " mafias " locales), y compris dans les zones où le réseau municipal n'est pas encore présent. Ils dénoncent alors en chœur les prix élevés pratiqués et les profits réalisés par ces vendeurs informels aux dépens des plus faibles, des " marginalisés ". D'après leurs analyses, l'absence du service officiel dans ces quartiers n'en serait qu'une conséquence, du fait du vandalisme par ces " organisations illégales ». Au départ, il m'était d'ailleurs fort difficile de critiquer le discours humaniste de mon tuteur dont la bonne volonté est sûrement sincère. Mais au-delà, c'est bien une conception capitaliste de l'eau comme ressource économique qui est mise en œuvre, participant à la diffusion des processus marchands dans la société et produisant de nouvelles inégalités intra-urbaines. Entre l'assimilation nécessaire du point de vue de l'entreprise et l'analyse des rapports de domination en œuvre dans l'accès à l'eau, la démarche de la géographie critique s'est imposée comme moyen de faire apparaître les systèmes de relations qui se construisent du fait des failles de ce service et de la structuration d'organisations parallèles pour en pallier les lacunes.

Ainsi est née ma réflexion sur les perceptions des inégalités, les discours de la pauvreté et sur leurs interrelations. Cette impossibilité de détourner mon regard de ces disparités et la volonté de comprendre le maintien de la pauvreté dans cette ville riche, témoin de la réussite économique d'un pays plein de ressources sont ainsi les moteurs de ce choix de recherche permettant d'interroger l'éloignement identitaire qui s'impose avec tous ceux que je fréquente là-bas. De la découverte d'un lieu de manière conjoncturelle s'est élaboré un questionnement constituant alors un terrain.

\section{La construction d'une démarche critique de recherche}

Pour répondre à mon incompréhension quant à l'incapacité d'une ville riche à répondre aux besoins fondamentaux de ses résidents et à ma révolte personnelle face aux insuffisances des initiatives menées au nom du traitement de la pauvreté, l'analyse critique de ces actions à partir de l'entrée spatiale, comme une clef de compréhension de la diversité des rapports politiques et sociaux, m'est apparue pertinente pour mettre en lumière les limites structurelles et politiques de ces actions.

A cette fin, c'est ma démarche de chercheuse qu'il m'a fallu construire afin de déceler les perceptions de tous les acteurs, populations, gestionnaires ou décideurs. C'est donc l'observation et l'entretien qui doivent être au cœur de ma méthodologie en intégrant l'éloignement culturel comme une donnée fondamentale et en même temps un biais dans la production des résultats. C'était pour moi le meilleur moyen de mettre en perspective des données statistiques officielles abondantes en Indonésie qui, de par leur questionnement, plaquent des conceptions du Nord sur leur pays. Leur objectif est en effet de répondre aux critères du développement humain de l'ONU et à ceux de la Banque mondiale. Le choix de cette méthode qualitative est donc un moyen d'éviter une nouvelle fois la reproduction de ces biais et d'affirmer une approche différente face à l'accumulation des recensements à 
Jakarta. Cette approche méthodologique s'explique aussi par le contexte de la recherche liée à mon acceptation dans les quartiers étudiés ${ }^{9}$. Je n'insisterai pas sur la question évidente et permanente d'un chercheur occidental dans une ville du Sud, régulièrement évoquée et traitée, notamment dans le premier numéro des Carnets par Pauline Guinard (Guinard, 2010). En effet, l'idée d'assimilation de ma personne dans des quartiers défavorisés n'est pas pertinente : ce n'est pas parce que l'on vit comme un pauvre qu'on est pauvre et qu'on en éprouve les conséquences. II faut donc se faire accepter avec nos différences, ce que l'on projette.

Le véritable défi a finalement été d'essayer de changer d'image. Comment ne pas passer pour une touriste ${ }^{10}$ qui vient regarder le pittoresque bidonville (un Indonésien organise justement ces tourist tours dans le quartier pour des occidentaux) alors que j'utilise le même " attirail " visible comme l'appareil photo ; comment ne pas être simplement un " porte monnaie » pour dépanner des personnes qui sont, en effet, dans la misère la plus profonde ; comment ne pas être non plus une ONG qui pourrait apporter éventuellement un changement dans cette communauté ; de l'autre côté, comment, moi, puis-je venir observer ces situations sans honte, sans voyeurisme, sans culpabiliser de leur prendre du temps en discutant avec eux alors que chaque minute peut être comptée comme un revenu (récupération des déchets souvent) ; et enfin, comment leur faire accepter cette discussion : Ne pas imposer une image et éviter ces images-là afin d'avoir une démarche acceptable et identifiable pour chacun.

II m'a donc fallu mettre au jour des aspects de mon identité que je n'estimais pas être publics : une religion (impensable de ne pas en avoir en Indonésie ${ }^{11}$ ), une vie familiale, en plus de mon origine alors qu'au départ tous ces éléments étaient des biais méthodologiques que je ne voulais pas ajouter à un statut déjà lourd de perceptions. Sans compter que moimême, je ne concevais pas l'idée que mes croyances et mon état marital pourraient participer à la mise en conformité de ma personne avec les catégories sociales. Et finalement, $c^{\prime}$ est ce qui a permis la réalisation de ces entretiens : pour avoir de l'information sur leur vie, il faut leur en donner sur la mienne. L'échange est là, l'information est là. C'est un besoin de savoir à qui l'on s'adresse et satisfaire une forme de curiosité. Après une question, la réciproque est souvent de mise. Il y a souvent une volonté de comparaison des situations avec celle de mon pays d'origine : " et comment cela se passe-t-il là bas ? "

En parallèle, dans ma démarche d'acceptation par les familles et de conviction personnelle, j'ai pris l'initiative de participer à l'enseignement dans une petite école informelle. Cela a eu beaucoup plus d'impact que je ne l'aurai pensé. En plus d'être connue par les mères et les enfants, c'était pour moi le moyen de leur apporter quelque chose d'essentiel (ou que je considère comme tel), qui ne s'échange pas contre de l'argent

\footnotetext{
9 Les tentatives d'un questionnaire ne se sont pas révélées pertinentes pour aborder les perceptions et pratiques spatiales des personnes en situation de pauvreté à Jakarta. C'était encore moins évident pour prendre en compte les rapports sociaux en place dans les quartiers liés aux positions de pouvoir et à l'exercice de la domination.

${ }^{10}$ Le problème pour moi n'est pas d'être ou non une touriste dans un pays étranger ni de dévaloriser la démarche du touriste. L'enjeu est de ne pas être perçue comme telle dans le quartier par les habitants. L'assimilation possible de ma personne à un touriste aurait des retombées sur la possibilité de faire mes enquêtes et observations, car, face aux touristes, j'ai observé une accentuation des comportements misérabilistes par la population elle-même, en particulier des enfants, qui demandent alors de l'argent " pour aller à l'école demain ". II ne m'était pas possible de mettre en place de telles relations avec eux au quotidien.

${ }^{11}$ Cet aspect est en effet un élément obligatoire qui figure sur la carte d'identité de tous les Indonésiens.
} 
(expliquant sûrement le fait qu'on ne m'en ait jamais demandé), de faire partie de leur quotidien dans lequel je ne suis ni oubliée, ni invisible, mais dans lequel il n'est plus étonnant de me voir ${ }^{12}$. C'était aussi l'occasion de $m^{\prime}$ investir dans un projet dans lequel je crois personnellement et le moyen d'accéder à d'autres informations que celles que peuvent obtenir des Indonésiens, du fait de la perception de ma personne comme différente, extérieure mais pas étrangère à leur quotidien.

De plus, la présence quotidienne sur le terrain permet de prendre en compte la diversité de la population d'un quartier (dont sa qualification de pauvre ou d'informel masque une réalité bien plus complexe) et de dépasser les contraintes de l'altérité. Des observations et des entretiens, l'enjeu est de faire apparaître les faits urbains à partir des situations individuelles. En fait, c'est bien nos différences qui permettent de faire apparaître toutes les discontinuités intra-urbaines, voire les fragmentations qui limitent l'accès à la ville à une partie de la population. Expérimenter la ville avec eux en circulant dans le quartier et les alentours à travers les espaces publics (ou qui apparaissent comme tels), les transports, les services me montre des limites qu'ils ont que je n'ai pas.

Alors que je rentrais chez moi en fin de journée, un petit groupe d'enfants auquel j'enseigne m'emboite le pas. Je ne réfléchis plus au trajet que j'effectue quotidiennement: je traverse la voie ferrée et me dirige vers la route principale afin de prendre le bus. Le chemin le plus court me fait passer par le bord du marché et j'entre dans le centre commercial voisin ; l'arrêt est de l'autre côté. Deux enfants s'arrêtent net, l'un part en courant sans rien dire. Ce dernier a voulu éviter un agent de sécurité qui l'a déjà identifié comme un anak payung : un enfant parapluie. Lorsqu'il pleut, il se précipite à l'entrée avec de nombreux camarades et des parapluies pour abriter les chalands contre une pièce. Le garde (un habitant du même quartier d'ailleurs) lui, a comme mission de maintenir éloigner la présence de ces " emplois informels " qui attirent des pauvres trop près du mall ${ }^{13}$. De même, il nous rappelle qu'il est formellement interdit d'entrer pieds-nus pour le garçon à côté de moi.

Ainsi émergent les barrières invisibles différenciées selon la population dues aux distances, aux interdictions, et parfois aussi aux coûts qui imposent des restrictions d'accès à l'espace urbain aux plus défavorisés. La ligne de rupture qui sépare le monde informel du formel perd ainsi de son sens lorsqu'on la confronte au terrain montrant ainsi les limites de cette " catégorie » de l'informalité. Les liens et interrelations sont permanents dans l'espace urbain. Mais l'intégration de ces normes - ou plutôt leur internalisation - par la population et les services produit de nouvelles formes de domination que l'analyse critique ${ }^{14} \mathrm{~m}^{\prime} \mathrm{a}$

\footnotetext{
${ }^{12}$ L'enseignement chaque matin a permis la mise en place d'une régularité de ma présence dans le quartier ce qui a facilité la demande d'entretiens auprès des ménages et je suppose aussi l'acceptation de ces discussions permettant l'instauration d'une relation de confiance et de réciprocité. D'un point de vue méthodologique, cela m'a permis aussi d'identifier les familles dont les enfants n'ont pas accès à l'école formelle.

${ }^{13}$ Propos issus de mes entretiens, juillet 2011.

${ }^{14}$ Cécile Gintrac (2012) présente une synthèse de la pensée critique. Elle met ainsi en valeur la combinaison de l'analyse évaluative des catégories avec l'acception marxienne qui cherche à " mettre au jour les formes cachées de domination et d'exploitation » (Wacquant, 2001).
} 
permis de déceler et de comprendre. Les concepts de "droit à la ville » (H. Lefebvre, 1968) et d'injustice spatiale sont ainsi assez opératoires pour faire apparaître ces rapports de force à l'intérieur de la population urbaine et en dépassant l'opposition riche/pauvre, formel/informel. Bien qu'il faille intégrer dans nos réflexions ces représentations binaires qui dominent les perceptions des acteurs, il nous faut faire apparaître les implications de ces catégories d'appréhension des relations sociales. Ainsi, la démarche critique nous permet de mettre en valeur ces conceptions duales: de l'analyse qui en est faite par les acteurs découlent des actions spécifiques qui ne remettent pas en cause ces dualités; mais cette analyse ne rend pas compte de la complexité des forces qui participent à la production de la ville, ce que le chercheur se doit alors de mettre en évidence.

Enfin, mon statut de chercheuse a poussé certains à vouloir instrumentaliser mon travail, utiliser mes résultats voire les influencer afin d'assurer la promotion de leur action. Que ce soit des ONG, des entreprises ou des opérateurs de politiques publiques, ma présence était un moyen de valoriser leur action voire de la légitimer. Ainsi certains ont cherché à limiter ou plutôt à encadrer mon travail d'enquête de différentes manières : au nom de la protection de ma personne et pour me faciliter le travail, un membre d'une ONG a cherché à me diriger vers des personnes " de confiance ", "qui vont me parler " qui étaient ainsi en mesure de montrer le bon travail qu'ils ont effectué dans le quartier. Des fonctionnaires et élus locaux ont voulu m'accompagner sur le terrain et être présents lors des entretiens et autres échanges informels. Que ces pratiques soient parties d'un bon sentiment n'est peut être pas inconcevable mais la réalité est que ce mode de fonctionnement impose un discours spécifique aux interviewés qui se plient plus ou moins consciemment au rapport de domination qu'exerce cet individu par sa présence. Ce dernier le met en œuvre grâce à son réseau d'interconnaissances et son rôle dans l'organisation sociale et spatiale du quartier (en tant que chef de quartier, gestionnaire des politiques publiques locales, ou généreux donateur qui aide financièrement mais ponctuellement les personnes en difficulté). Sortir de ces pratiques n'est d'ailleurs pas évident, et ma simple présentation aux côtés de ces acteurs participe aux projections que les habitants se font. Dans le même temps, il m'était impossible d'entrer dans un quartier sans respecter les coutumes locales qui veulent que l'on aille se présenter (avec nos autorisations) aux chefs de quartiers et personnes de confiance de la communauté (cadres sociaux plus ou moins officieux, chefs religieux, ...), sans quoi les habitants restent soupçonneux ou simplement inquiets de nos intentions. L'analyse critique de ces entretiens et de la perception de ces volontés de manipulation montre ainsi l'enjeu de ces quartiers pauvres dans la ville et l'importance des rapports de domination en œuvre dans la question du traitement de la pauvreté à Jakarta. 
Conclusion : La recherche critique comme réponse à notre utilité sociale : la quête d'une posture éthique

Entre la mise en lumière des structures sociales, des rapports de domination qui s'expriment frisant souvent la dénonciation de politiques inadaptées et la non-intervention du chercheur dans ces politiques par choix méthodologique pour les analyser, comment accepter notre participation au système quand on ne le combat pas ? La rédaction d'une thèse qui sera peu lue globalement ou d'articles scientifiques peu accessibles et les quelques interactions avec les décideurs sont-ils suffisants pour un chercheur face à l'urgence des besoins et la précarité extrême de ceux qu'il observe? Sans condescendance ni misérabilisme, comment sortir de cette séparation entre le savant et le politique, simplement en tant que citoyen acteur du lieu où il vit, travaille (au moins une partie de l'année) et interagit ? Don Mitchell par exemple est notamment revenu ${ }^{15}$ sur la construction de son positionnement critique en lien avec le contexte de la production de ses recherches. L'enjeu est bien ici, à partir d'expérience de terrain, de comprendre les rapports de domination en œuvre qui vont bien au-delà de relations interpersonnelles. A partir de ces réflexions, c'est bien la critique de l'Etat qui est en jeu (Sassen, 2009).

Au-delà, le terrain dépasse la production du savoir et ses conditions. Ce sont les échanges et les rencontres avec les personnes qui en font la richesse. L'accueil, les sourires et le regard de reconnaissance réciproque montrent l'acceptation de l'autre tel qu'il est.

\section{Bibliographie}

Barley N. (1988), L'anthropologie n'est pas un sport dangereux, Paris, Editions Payot, 288p.

Calbérac Y. (2011), "Le terrain des géographes est-il un terrain géographique ? Le terrain d'un épistémologue ", Carnets de géographes, n², Varia. 6 p.

Collignon B. (2008), "Note sur les fondements des postcolonial studies ", EchoGéo [En ligne], 1 | 2007, mis en ligne le 06 mars 2008, URL : http://echogeo.revues.org/2089 8 p.

Gintrac C. (2012), "Géographie critique, Géographie radicale: Comment nommer la géographie engagée ? " Carnets de géographes, n4 Géographies critiques. 13 p.

Guinard P (2010), «Regard et place d'une chercheuse blanche dans les espaces publics de Johannesburg ", Carnets de Géographes, $n^{\circ} 1$, Varia. 7 p.

Hancock C. (2007), " "Délivrez nous de l'exotisme" : quelques réflexions sur des impensés de la recherche géographique sur les Suds (et les Nords) ». In : Gervais-Lambony P. (ed.), Landy F. (ed.) On dirait le Sud .... Autrepart, 2007, (41), p. 69-81.

Lefebvre H. (1968), Le droit à la ville, Paris, Éd. Anthropos, 166 p.

Pillow W. (2003), "Confession, catharsis or cure? Rethinking the uses of reflexivity as methodological power qualitative research", Qualitative Studies in Education, vol 16. p. 175196

Renganathan S. (2009), "Exploring the Researcher-Participant Relationship in a multiethnic, multicultural and multilingual context through reflexivity", Qualitative Research Journal, vol9 n². p. 3-17

\footnotetext{
${ }^{15}$ Conférence plénière du colloque "Espace et rapports sociaux de domination : chantiers de recherche » à Marne la Vallée : « On Becoming and Being a Radical Geographer in the United States », septembre 2012.
} 
Revol C. (2011), "Lefebvrian English-speaking reception of " Right to the city »: transpositions and present meaning", communication à ESA Conference, Genève, septembre 2011 (non publié)

Sassen S. (2009), Critique de l'Etat - Territoire, Autorité et Droits de l'époque médiévale à nos jours, Demopolis, Monde diplomatique, 474p.

Silver C. (2008), Planning the megacity: Jakarta in the twentieth century, Planning, history, and the environment series, Routledge, 262p.

Volvey A. (2003), "Terrain » in Levy J et Lussault M. (dir.), Dictionnaire de la géographie et de l'espace des sociétés, Paris, Belin p 904-906. 